\title{
Properties of the C-terminal domain of HlyIICTD suggest that $B$. cereus HlyII is a representative potential member of trimeric autotransporter adhesins among gram-positive bacteria
}

\author{
Siunov A.V. \\ G. K. Skryabin Institute of \\ Biochemistry and physiology of \\ microorganisms RAS, \\ Pushchino, Russia \\ Nagel A.S. \\ G. K. Skryabin Institute of \\ Biochemistry and physiology of \\ microorganisms RAS, \\ Pushchino, Russia \\ Andreeva-Kovalevskaya Z.I. \\ G. K. Skryabin Institute of \\ Biochemistry and physiology of \\ microorganisms RAS, \\ Pushchino, Russia \\ Zamyatina A.V. \\ Pushchino State Institute of Natural \\ Sciences, Pushchino, Russia \\ Pushchino Branch, Shemyakin- \\ Ovchinnikov Institute of Bioorganic \\ Chemistry, Pushchino, Moscow, Russia \\ Rudenko N.V. \\ Pushchino State Institute of Natural \\ Sciences, Pushchino, Russia \\ Pushchino Branch, Shemyakin- \\ Ovchinnikov Institute of Bioorganic \\ Chemistry, Pushchino, Moscow, Russia \\ Karatovskaya A.P. \\ Pushchino Branch, Shemyakin- \\ Ovchinnikov Institute of Bioorganic \\ Chemistry, Pushchino, Moscow, Russia \\ Borisova M.P. \\ Institute of Theoretical and \\ Experimental Biophysics, Russian \\ Academy of Sciences, \\ Pushchino, Russia
}

\author{
Brovko F.A \\ Pushchino State Institute of Natural \\ Sciences, Pushchino, Russia \\ Pushchino Branch, Shemyakin- \\ Ovchinnikov Institute of Bioorganic \\ Chemistry, Pushchino, Moscow, Russia \\ Salyamov V.I. \\ G. K. Skryabin Institute of \\ Biochemistry and physiology of \\ microorganisms RAS, \\ Pushchino, Russia
}

Solonin A.S.

G. K. Skryabin Institute of

Biochemistry and physiology of microorganisms RAS,

Pushchino, Russia

Pushchino State Institute of Natural Sciences,

Pushchino, Russia

\footnotetext{
Key-words - hemolysin, autotransporter proteins, in pore formation
}

Hemolysin II of B. cereus sensu lato is synthesized in a bacterial cell in the form of a water-soluble secreted monomer and penetrates into eukaryotic membranes forms weakly anion-selective pores, the radius of which varies from 0.6 to $0.8 \mathrm{~nm}$ in both natural and model erythrocyte membranes [1]. In a mature state, this toxin is the closest homologue of $S$. aureus alpha toxin (38\% amino acids identity). The HlyII protein has a C-terminal extension not previously described in this class of toxins, including 94 amino acid residues designated as HlyIICTD (C-terminal domain) [2]. Removal of HlyIICTD from HlyII does not allow transfer of the deletion variant to $E$. coli cells, possibly due to the attack of the bacterial membrane by the deletion toxin during its accumulation in the periplasm. Additional deletion of the signal peptide, which excludes the penetration of the protein into the periplasm, provides for the cloning of this gene with two deletions in E. coli cells. The deletion variant of hemolysin II has a reduced hemolytic activity. Using monoclonal antibodies against recombinant HlyIICTD, HlyIICTD showed the possibility to bind effectively to red blood cells [3]. Purified HlyIICTD preparations are capable to trimerization in the presence of
4M urea and, forming a possibly beta-barrel-like structure, integrate into the artificial bilayer membrane with the formation of pores. The paper presents the current-voltage characteristic of these pores. Such structures are characteristic of trimeric autotransporter proteins. In this case, the secreted full-sized monomeric form of hemolysin II acts as a passenger, and HlyIICTD acts as an element involved in adhesion. The materials presented in this paper demonstrate the possible functional role of HlyIICTD hemolysin II both in pore formation and in the secretion of toxin from a bacterial cell, suggesting that hemolysin II may belong to trimeric autotransporter proteins - the first case of the description of this family of molecules among grampositive microorganisms.

\section{REFERENCES}

[1] Andreeva Z.I., Nesterenko V.F., Fomkina M.G., TernovskyV.I., Suzina N.E., Bakulina A.Y., Solonin A.S., Sineva E.V. // Biochim. Biophys. Acta. 2007. V.1768. P.253-263.

[2] Baida G., Budarina Z. I., Kuzmin N.P., Solonin A.S. // FEMS Microbiol. Lett. 1999. V.180. P.7-14.

[3] Rudenko N. V., Karatovskaya A. P., Zamyatina A. V., Siunov A. V., Andreeva-Kovalevskaya Zh. I., Nagel A. S., Brovko F. A., and A. S. Solonin // Russian Journal of Bioorganic Chemistry, 2020, Vol. 46, No. 3, pp. 321-326. 